\title{
MedienPädagogik
}

Zeitschrift für Theorie und Praxis der Medienbildung

www.medienpaed.com

ISSN 1424-3636

Themenheft Nr. 37: Medienpädagogik als Schlüsseldisziplin in einer mediatisierten Welt. Perspektiven aus Theorie, Empirie und Praxis Herausgegeben von Henrike Friedrichs-Liesenkötter, Lara Gerhardts, Anna-Maria Kamin und Sonja Kröger

\section{Medienpädagogische Praxisarbeit als Pionierin für die Verbreitung von OER}

\author{
Charlotte Echterhoff und Sonja Kröger
}

\section{Zusammenfassung}

In dem Beitrag plädieren die Autorinnen dafür, dass eine Medienpädagogik, die sich als Schlüsseldisziplin in einer digitalisierten Welt versteht, Vorbildfunktion übernehmen kann: Um den Kulturwandel in der Bildungslandschaft stärker zu fördern, kann die Medienpädagogik die Idee offener Bildung und die Verbreitung von Open Educational Resources (OER) noch nachhaltiger in Praxis und Forschung etablieren. Es wird zunächst der bildungspolitische Hintergrund von Offenen Bildungsmaterialien dargestellt und international verordnet. Darüber hinaus werden die Potenziale von OER für die medienpädagogische Praxisarbeit aufgezeigt. Die Relevanz von OER zeigt sich in traditionellen Schulpraktiken, die noch nicht primär Kreativität und Kooperation lehren - obwohl dies relevante Kompetenzen für die Zukunft sind. Die Autorinnen zeigen, dass neue Formen des Lehrens und Lernens aufgrund fortschreitender Digitalisierung im Bildungsbereich dringender denn je sind. OER werden vor dem Hintergrund als ein Vehikel für den notwendigen Kulturwandel beschrieben. Die aktuellen bildungspolitischen Entwicklungen legen nahe, dass jetzt der richtige Zeitpunkt ist, um den OER-Gedanken noch nachhaltiger in der medienpädagogischen Praxisarbeit zu verankern und somit verstärkt innerhalb der Medienpädagogik und anderen Disziplinen zu verbreiten. 


\title{
Practical media education work as a model for the dissemination of OER
}

\begin{abstract}
The authors argue that media pedagogy - as a key discipline in a digitalized world - can act as a role model. Promoting a cultural change in the educational landscape, media pedagogy can thus establish the idea of open education and the spread of Open Educational Resources (OER) even more in practice and research. After describing the educational background of open educational resources in the international framework, the potential of OER for practical media education work is analysed. OER's relevance is shown in traditional school practice which is not yet primarily teaching creativity and cooperation - skills needed for the future. The authors show that new forms of teaching and learning are more urgent than ever due to advancing digitalization in the field of education. Because of that, OER can become the vehicle for the necessary cultural change. Moreover, current developments in education policy suggest that now is the right time to firmly anchor the concept of OER in media pedagogy's practical application and thus to spread it more widely within media pedagogy and other disciplines.
\end{abstract}

\section{Digitalisierung, Kulturwandel, Bildung - inter- national und in Deutschland}

Als Open Educational Resources (OER) gelten «Lern-, Lehr- und Forschungsmaterialien, in jedem Format und Medium, die gemeinfrei sind oder urheberrechtlich geschützt und unter einer offenen Lizenz veröffentlicht sind, wodurch kostenloser Zugang, Weiterverwendung, Nutzung zu beliebigen Zwecken, Bearbeitung und Weiterverbreitung durch Andere erlaubt wird» (UNESCO 2019, 3). Eine solche Lizenzierung erfolgt bspw. mit den Creative Commons-Lizenzen: Mit ihnen gewähren Urheberinnen und Urheber Dritten die Nutzungsrechte an ihrem Werk - was die Nennung des Urhebers oder der Urheberin nicht ausschliesst. 
Als supranationaler Player setzt die UNESCO das Thema Open Educational Resources (OER) seit einigen Jahren auf die Tagesordnung. ${ }^{1}$ Auch in Deutschland sind OER Teil der politischen Agenda. Der bestehende politische Aktionswille spiegelt sich im Koalitionsvertrag wider, in dem OER in den Zusammenhang digitaler Kompetenzen in einer modernen Wissensgesellschaft gestellt werden (Koalitionsvertrag vom 12. März 2018, 40). Bereits im Jahr 2016 widmete sich das Bundesministerium für Bildung und Forschung (BMBF) auf Grundlage eines Bund-Länder-Beschlusses dem Thema OER. Es wurden neben einer OER-Informationsstelle weitere 23 Projekte gefördert, in denen Multiplikatorinnen und Multiplikatoren aus den verschiedenen Bildungsbereichen für die Erstellung und Nutzung von OER sensibilisiert und qualifiziert wurden.

Die Prämisse, dass ein 〈Mehr an Bildung〉 nur vorteilhaft sein kann, begründete einst die Diskussion um Open Educational Resources. Es ging darum, technologische Möglichkeiten zu nutzen, um Bildungsmaterial für Entwicklungsländer einfach und günstig bereitzustellen (Muuß-Merholz und Schaumberg 2014, 12). Heute werden OER folglich in Zusammenhang mit Ziel 4 der globalen Ziele für nachhaltige Entwicklung gesehen: «Inklusive, gerechte und hochwertige Bildung gewährleisten und Möglichkeiten des lebenslangen Lernens für alle fördern» (BMZ 2017, 8). Die UNESCO schreibt OER eine Chance zur Förderung von Wissensgesellschaften und zur Förderung von Bildung für alle Menschen weltweit zu. Aufgrund der

1 Die UNESCO prägte den Begriff 〈Open Educational Resources〉 (kurz: OER). Mit der Pariser Erklärung (2012) wird als Abschluss der ersten UNESCO-Weltkonferenz zu OER den Mitgliedstaaten die Förderung von OER empfohlen. Seitdem gewinnen OER als Thema international stark an Bedeutung. So verabschiedete die Europäische Kommission im Jahr 2013 eine Mitteilung, in der sie in dem Zusammenhang das Ziel beschreibt Bildung zu öffnen: Innovatives Lehren und Lernen soll für alle mithilfe neuer Technologien und frei zugänglicher Lehr- und Lernmaterialien möglich werden (Weiterführende Informationen dazu unter https://eur-lex.europa.eu/legal-content/DE/TXT/PDF/?uri =CELEX:52013DC0654\&from=EN). Als Ergebnis der zweiten UNESCO Weltkonferenz zu OER wurde 2017 der 〈Ljubljana OER Action Plan〉 verabschiedet, in dem weiterer Handlungsbedarf beschrieben wird (siehe: https://en.unesco. org/sites/default/files/ljubljana_oer_action_plan_2017.pdf). Zusätzlich wurde im November 2019 in Paris eine UNESCO-Empfehlung verabschiedet, die eine verbindlichere Form der Übereinkunft auf UNESCO-Ebene darstellt und für die Mitgliedstaaten mit mehr Pflichten verbunden ist, als es die 〈Erklärung〉 bisher war. 
den OER innewohnenden Zieldimensionen von Offenheit, Vervielfältigung, Verwendung, Verarbeitung und Verbreitung verfügen OER über das Potenzial, Bildungsqualität zu verbessern sowie Dialog, Verbreitung von Wissen und ¿Capacity Building〉 zu fördern. Damit leisten sie laut UNESCO einen Beitrag zur Umsetzung der Bildungsagenda 2030 (siehe weiterführend dazu: https://www.unesco.de/bildung/bildungsagenda-2030).

Welche Kompetenzen benötigen zukünftige Generationen? Diese Frage wird mehr denn je diskutiert; spätestens seitdem das Smartphone zu einem allgegenwärtigen Begleiter für Jugendliche geworden ist und somit jede und jeder quasi einen endlosen Wissensfundus mit sich trägt. Es ist evident, dass nicht das Faktenwissen allein von Bedeutung sein kann, denn die digitalen Speicherkapazitäten werden die individuellen Wissenskapazitäten beständig überbieten. Das derzeitige Schul- und Bildungssystem zielt vornehmlich auf individuelle Leistungsmessung und Selektion ab. Es erscheint somit nicht nur im Hinblick auf eine nachindustrielle Gesellschaft (Baethge 2006, 26) als überholt, sondern auch angesichts der Digitalisierung in allen Lebens- und Arbeitsbereichen. Dies wird auch offensichtlich, weil digitale Medien wie Tablets, Smartphones und Whiteboards verstärkt Einzug in deutsche Schulklassen halten. Digitale Medien bieten zwar grosses Potential zur Gestaltung neuer Lern- und Lehrprozesse. Für einen sinnvollen Einsatz im Sinne individueller Förderung von Schülerinnen und Schülern erfordert der Einsatz digitaler Medien andere Kompetenzen von Lehrenden und Lernenden. Dazu zählen u.a. auch die genannten sozialen Fähigkeiten Kooperation und Kreativität. Als eine erste politische Antwort zur Neuausrichtung der Bildung in einer digitalen Welt ist diesbezüglich die KMK Strategie (2016) zu sehen. Der notwendige 〈Kulturwandel〉 wird im Bildungsbereich in Deutschland darüber hinaus unter dem Stichwort 〈zeitgemäße Bildung〉 diskutiert. Hier stehen neue Formen des kooperativen und kreativen Arbeitens im Zentrum, die sich um Offenheit statt um Abgrenzung bemühen. Es geht um lernendenzentrierten Unterricht, um Partizipation (Mayrberger 2014) sowie darum, jenseits der kognitiven die sozialen Aspekte des Lernens zu betonen (vgl. Deimann 2012, 17). Aus mediendidaktischer Sicht können digitale Medien diesen Systemwechsel befördern und der Öffnung von Unterricht aufgrund ihrer technischen Gegebenheiten zutragen. Sollen also solche Kompetenzen für die Zukunft 
erlernt werden, können OER dabei als ein wichtiges Vehikel für den Kulturwandel in der Bildung fungieren.

Ein Beispiel dafür ist der tertiäre Bildungsbereich: OER sind im Rahmen der Open-Bewegung zunächst in den Hochschulen als innovatives Konzept integriert worden (Zauchner und Baumgartner 2007). Trotz möglicher Synergieeffekte im Zusammenhang mit Diskussionen um bspw. Open Access haben sich OER nach wie vor in diesem Bildungsbereich nicht in der Breite durchgesetzt. Gründe dafür liegen möglicherweise in der primär durch Konkurrenz und weniger durch Kooperation geprägten Hochschul- und Wissenschaftscommunity. Zudem scheint ein Bewusstsein für einen möglichen Mehrwert von OER bei vielen Akteuren in der Hochschullandschaft noch nicht ausreichend ausgeprägt; dies kann auch auf fehlende Qualifikations- und Unterstützungsangebote zurückzuführen sein. Unabhängig vom Bildungsbereich trägt ggf. auch ein noch nicht bestehender OER-Metadatenstandard dazu bei, dass die Chancen und Potenziale von OER in Deutschland noch nicht flächendeckend genutzt werden (vgl. Deimann, Neumann, und Muuß-Merholz 2015, 56-60). Es ist dennoch folgerichtig, dass im Rahmen der Diskussionen um den 〈DigitalPakt Schule〉 ${ }^{2}$ das Thema OER aktuell explizit für den schulischen Bildungsbereich aufgewertet wird. Dahinter steht die Hoffnung, dass mit den Mitteln des 〈DigitalPakt Schule> keine reine Technikförderung erfolgt, sondern - zumindest langfristig - durch den Einsatz digitaler Medien und Technologien neue Lehr- und Lernkonzepte Eingang in die schulische Praxis erhalten. Die Entwicklung neuer Lehr- und Lernkonzepte impliziert dabei, dass das Ziel von OER nicht sein kann, das scheinbar 〈perfekte) Arbeitsblatt für einen 45-minütigen Fachunterricht zu liefern. Vielmehr sind die Ziele jenseits der didaktischen Lernendenzentrierung zu finden: etwa die Öffnung von Themen, das Verlassen des Klassenraums, das Aufbrechen des Fächerkorsetts und die Aufhebung starrer Zeitvorgaben.

2 Der DigitalPakt Schule ist mit 5,5 Milliarden Euro das bisher grösste Förderund Investitionsprogramm, das das BMBF auf den Weg gebracht hat. Mit dem DigitalPakt Schule wollen Bund und Länder gemeinsam flächendeckend in allen Schulen in Deutschland eine digitale Infrastruktur einrichten. Während der Bund die technische Infrastruktur finanziert, haben sich die Länder dazu verpflichtet sicherzustellen, dass Lehrpersonen entsprechend qualifiziert werden. 
Das Potential von OER im schulischen Bereich liegt zum einen in neuen didaktischen Möglichkeiten auf Ebene des Lernens, des Unterrichts und der Zusammenarbeit. Daneben eröffnet die mit der Lizensierung gegebene Rechtssicherheit digitaler Materialien neue Wege. Kopierte Arbeitsblätter in Deutschlands Zimmern des Lehrpersonals stehen in der Regel nicht mit den Urheber- und Nutzungsrechten von bspw. Verlagsprodukten in Einklang. Dieser Status Quo könnte mit der konsequenten Nutzung von offenlizensierten Bildungsmaterialien in ein rechtskonformes Modell transformiert werden. Zwar gelten Bildungsmaterialien umgangssprachlich bereits als 〈gemeinfrei〉 und die gelebte Praxis im digitalen Zeitalter zeichnet sich durch Downloaden, Nutzen, Kopieren, Verändern und Weitergeben aus. Erst mit einer entsprechenden Lizensierung würde der rechtliche Unterbau für diese Praxis geliefert und Rechtssicherheit hergestellt (MuußMerholz und Schaumberg 2014, 43). ${ }^{3}$

Mit den Stichworten 〈Kooperation〉, 〈Kreativität〉 und 〈Teilen〉 werden offene Bildungsmaterialien in Zusammenhang von zeitgemässen Lehr-/ Lernkonzepten gestellt (vgl. insbesondere zum Begriff des Teilens: Bock und Tribukait 2019). In den bisherigen Debatten stehen allerdings vornehmlich die didaktischen Möglichkeiten im Vordergrund: etwa, dass aus bestehenden OER durch geringfügige Anpassungen seitens der Lehrkräfte individualisierte Lernmaterialien für binnendifferenzierte Lerngruppen erstellt werden können (Muuß-Merholz und Schaumberg 2014, 39-40).

Open Educational Resources stehen daher auf der einen Seite in engem Zusammenhang mit digitalen Formaten und dem aufgrund der Digitalisierung vereinfachten Austausch von digitalen Materialien. Auf der anderen Seite beinhalten OER explizit nicht nur digitale Materialien, denn: Aus der OER-Idee wird das Ideal freier Bildung aus dem gesamtgesellschaftlichen Nutzen möglichst weitverbreiteter, zugangsschrankenarmer und hochwertiger Bildung abgeleitet.

Die Arbeit mit den Materialien, der rechtlich zulässige Rahmen des Austauschs und der Weiterverwendung sind ein Aspekt des grossen Potentials von OER. Ein weiterer liegt in der Produktion von digitalen

3 Dieser Logik entsprechend existieren öffentlich-rechtliche Projekte, die digitale Medienprodukte unter einer freien Lizenz mit dem Ziel der schulischen Nachnutzung online verfügbar machen, wie etwa auf der Internetseite www. planetschule.de. 
Medienangeboten, die durch die Verwendung von offen lizensierten Ausgangsmaterialien wesentlich vereinfacht wird. Wie die Beiträge in dem Heft 34 der 〈MedienPädagogik〉 zum Themenschwerpunkt OER aufzeigen, wird bislang noch wenig darüber diskutiert, wie das der OER-Idee zugrunde liegende Ideal freier Bildung gesamtgesellschaftlich stärker umgesetzt werden kann (u.a. Bock und Tribukait 2019, 62; Kerres 2019), um inklusive, gerechte und hochwertige Bildung zu gewährleisten und somit lebenslanges Lernen für alle zu ermöglichen. Offenkundig erscheint, dass die Diskussionen um Didaktik dafür noch zu kurz greifen.

\section{Potenziale von OER für die medienpädagogische Praxisarbeit}

Die Diskussion um OER thematisiert primär noch die Bildungsbereiche 〈Hochschule〉 (u.a. Deimann 2018; Bellinger und Mayrberger 2019; Missomelius 2019) und 〈Schule〉 (u.a. Bock und Tribukait 2019; Muuß-Merholz und Schaumberg 2014; Schön u. a. 2017). Im Bereich der Hochschule spielen neben OER auch andere 〈Open〉-Charakteristika eine Rolle, etwa die «Öffnung von Zugängen zu Informationen (Open Access) und das Überwinden von Grenzen beim Lernen (Open Education)» (Deimann 2012, 12). Im Bereich der Schule spielen neben der Diskussion um didaktische Potenziale (Mayrberger 2014) auch Fragen des Urheberrechts eine entscheidende Rolle (Erdsiek-Rave und John-Ohnesorg 2014). Auf der anderen Seite wird die Konvergenz der Systeme 〈Schule〉 und 〈OER〉 infrage gestellt, da die mit OER einhergehende 〈Kultur des Teilens〉 nicht mit dem in der Schule vorherrschenden Leistungsprinzip vereinbar scheint und auch der starre Zeitrahmen selbstverantwortliche und kooperative Formen des Lernens oftmals konterkariert (Bock und Tribukait 2019, 59).

Unabhängig von den wichtigen Bestrebungen, diverse Open-Bewegungen zu verbinden (Kerres 2019), plädiert dieser Artikel dafür, neben den Bildungsbereichen 〈Hochschule〉 und 〈Schule〉, die offensichtlich mit erheblichen institutionellen Schranken zu kämpfen haben, die ausserschulische Bildungsarbeit und somit auch informelle Lernprozesse stärker in den Blick für das Potenzial von OER zu nehmen. Es handelt sich damit um ein Bildungsfeld, in dem die Medienpädagogik eine starke Tradition in 
Deutschland hat. Dies erscheint insofern konsequent, da OER, historisch betrachtet, auch die Entwicklung einer offenen Gesellschaft und Bildung forciert, in denen es «um mehr Transparenz, stärkere Teilhabe und verbesserte Zusammenarbeit innerhalb zentraler gesellschaftlicher Bereiche» (Deimann 2012, 4) geht.

Für die nachhaltige Verankerung von OER in der ausserschulischen Bildungsarbeit erscheint es wichtig, neben OER auf (Open Educational Practice> (OEP), also Einsatzszenarien zu OER, zu setzen. Aus mediendidaktischer Sicht sind OEP entscheidend, «in der die Lehrenden bzw. Anbietenden von OER und Lernenden gleichermaßen beteiligt sind - sei es im formalen oder eher informellen Bildungskontext» (Mayrberger 2014, 55). Genau hier können medienpädagogische Akteure in ausserschulischen Bildungsfeldern Pionierarbeit leisten und Angebote als Experimentierräume entwickeln, um das Innovationspotential von OER zu heben. Dies wäre eine angemessene Antwort der Medienpädagogik in der gegenwärtigen Situation, in der u.a. die Digitalisierung die Notwendigkeit eines Kulturwandels in der Bildung begründet und von Wissenschaft, Politik, Praxis und Wirtschaft Änderungen eingefordert werden. Während im schulischen Kontext der institutionelle Rahmen und «ideologische Machtverhältnisse» (Horndasch und Weichert 2014, 60) die Potenziale kooperativer Arbeit mit und an OER konterkarieren (Bock und Tribukait 2019, 61), kann ausserschulische Bildungsarbeit davon unabhängig neue Formen des Lernens in andere Bildungsbereiche verbreiten und das Gemeinschaftsprinzip befördern. Die dahinterliegende Hoffnung wäre, dass erprobte Best-Practice-Ideen eine grosse Strahlkraft entfalten und kurz- bis mittelfristig Innovationspotentiale auch für stärker formalisierte Lernorte aufzeigen.

Die inhaltliche Nähe der Medienpädagogik zu OER liegt unter anderem in der Methode der 〈Aktiven Medienarbeit) begründet. Ausgehend von handlungsorientierten Konzepten in der medienpädagogischen Praxisforschung ist die Aktive Medienarbeit, die methodisch auf der Projektarbeit nach Frey (1982) fusst, seit den 1980er Jahren von zentraler Bedeutung. Dabei zählt die Entwicklung von Medienkompetenz im Rahmen Aktiver Medienarbeit zum Bildungsprozess (Echterhoff 2012, 2). Mit Rückgriff auf Klafkis kategoriale Pädagogik, in der Bildung durch die Fähigkeit der Selbstbestimmung, die Solidaritätsfähigkeit und die Mitbestimmungsfähigkeit 
gefasst wird (Klafki 1996, 40), gilt Lernen somit als Interaktionsprozess. So zielen zentrale Aspekte dieser Methode auf weitestgehend ähnliche Ziele und Ideen ab, die bereits oben im Hinblick auf OER beschrieben wurden. Dabei stehen Handlungskompetenz und kommunikative Kompetenz im Fokus medienpädagogischer Praxisarbeit und beschreiben keinen statischen Zielzustand, sondern einen prozessualen, den es immer wieder neu zu erreichen gilt (Schell 2003, 64). Für den interaktiven Kommunikationsraum Internet berücksichtigt eine umfassende Handlungskompetenz nicht nur die passive Nutzung von Internetangeboten, sondern ebenso das eigenständige Erstellen von Inhalten. Im Zusammenhang mit Medienkompetenz (nach Baacke 1996) umfasst das übergeordnete Ziel der Mündigkeit aktive Teilhabe, da nur so Souveränität und Autonomie gegeben sind. Die gelebte Praxis einer solchen 〈aktiven Teilhabe〉 im Internet ist durch rechtswidriges Nutzen und Teilen urheberrechtlich geschützten Materials geprägt (etwa, wenn auf 〈Facebook〉 dazu aufgerufen wird, Bilder von Comichelden der Kindheit als Profilbild zu setzen). Dies liegt mitunter in der Interaktivität des Mediums begründet, welche aus technischer Sicht die Möglichkeit für Kollaboration stets mitdenkt. Sowohl der nationalen als auch der europäischen Rechtsprechung gelingt es nicht, mit Urheber- und Nutzungsrechten die volatile Welt des Digitalen angemessen zu beschreiben. Politische Abstimmungswege sowie das starre System 〈Recht〉 scheitern beispielsweise mit ihren Konzepten von Nationalstaaten und -grenzen (vgl. Urheberrechtsregelung auf europäischer Ebene).

OER bieten mit ihrer Lizenz einen praxisorientierten Bottom-Up-Ausweg, der sich als passgenauer als eine mögliche Top-Down-Regulierung erweist. Wird in der informellen Bildungsarbeit mit OER gearbeitet, so wird nicht nur für das Thema sensibilisiert, sondern auch eine zeitgemässe Lösung für die gelebte Kultur des Teilens im Internet aufgezeigt. Als Zielvorstellung werden OER der neue Standard im Internet, der den Kulturwandel in der Bildung dadurch manifestiert, dass CC-Lizenzen keine Zugangsschranken beinhalten und somit Kooperation, Kollaboration und Offenheit als Norm gesetzt werden. Das Selbstverständnis der medienpädagogischen Praxisarbeit, offene Bildungsressourcen für die Allgemeinheit zu generieren und zu nutzen kann dadurch gestärkt werden. 
Innerhalb der Lerntheorie kann Aktive Medienarbeit dem 〈Lernen durch Gestalten> (Stahl 2009) zugeordnet werden: Hier steht der aktive, selbstgesteuerte Wissenserwerb im Vordergrund, womit die Integration digitaler Medien in Lernszenarien ihre Vorteile erst dann entfaltet, wenn Lernende selbst digitale Medien gestalten (Damnik u. a. 2018). In der medienpädagogischen Praxis stehen der Methode, die die Weiternutzung von bestehenden digitalen Materialien für andere Kontexte vorsieht, oftmals urheberrechtliche Beschränkungen entgegen: Lernende durchforsten das Internet etwa auf brauchbares Video- oder Audiomaterial, um damit ein eigenes Produkt zu gestalten. Die benannte Problematik des Urheberrechts begründet den Mehrwert von OER in diesem Zusammenhang: Sie entsprechen ihrem Wesen nach der Praxis des Teilens im Internet. Wo derzeit primär in Grauzonen agiert wird, stellen die CC-Lizenzen ein zeitgemässes Konzept zur Verfügung, das es allen Nutzenden erlaubt, rechtssicher Material aus dem Internet weiter zu nutzen und selbst zu publizieren.

In der (formalen und informellen) Mediendidaktik ist nach Mayrberger neben 〈Interaktion〉 auch 〈Partizipation〉 von zentraler Bedeutung. Beim partizipativen Lernen geht es darum, dass auch die Gestaltung der Lernumgebungen mit den Lernenden zusammen geplant, umgesetzt und evaluiert wird (Mayrberger 2014, 53). Auch um diesen partizipativen Prozess einzuüben, erscheint die informelle Bildungsarbeit ein geeigneter Ort, wenn mit und an OER gearbeitet wird. Wenn die Gesamtheit verfügbarer OER von Lernenden genutzt werden kann, können sie selbst entscheiden, welches Format ein Endprodukt hat, auf welchem Weg, mit welcher Software, zu welchem Thema, in welcher Lerngruppe es erstellt wird.

Werden solche selbstbestimmten Lernprozesse in der informellen Bildungsarbeit bewusster eingeübt und standardmässig angeboten, werden Lernende diese Freiheiten auch in anderen Lernkontexten einfordern. Dies geschieht bereits massenhaft, wenn für Jugendliche 〈Youtube〉 zum «digitalen Kulturort» (Rat für Kulturelle Bildung e.V. 2019, 7) wird, den sie zum Lernen nutzen und selbst entscheiden, welcher Kanal für welches Thema am besten geeignet ist.

Der WebTalk (nachhörbar unter: https://open-educational-resources.de/besonderheiten-von-oer-der-medienpaedagogik-aufzeichnungdes-gmk-webtalks), der im November 2019 im Rahmen des 36. Forum 
Kommunikationskultur der Gesellschaft für Medienpädagogik und Kommunikationskultur e.V. (GMK) geführt wurde, zeigt auf, dass, obschon die Auseinandersetzung von Medienpädagogik und OER als «Kinderschuhe» betitelt wird, OER kein genuin neues Thema in der Medienpädagogik darstellen. So werden konkrete Beispiele für frei verfügbare Inhalte im medienpädagogischen Bereich genannt, anhand derer die durchaus langjährige Pioniertätigkeit medienpädagogischer Akteure im Bereich OER deutlich wird: zum einen der in der Community bekannte «Medienpädagogik Open-Praxisblog (www.medienpaedagogik-praxis.de), auf dem ein breites Spektrum an Themen, Inhalten und Materialien veröffentlicht wird. Zum anderen 〈Freesound〉 (www.freesound.org), ein Internetangebot, dass frei nutzbare Töne zur Verfügung stellt. Dass zum jetzigen Zeitpunkt eine noch stärkere Verschränkung von Medienpädagogik und OER thematisiert wird, verdeutlicht auch, dass die bildungspolitische Relevanz auf übergeordneter Ebene erkannt und damit begonnen wird, den Diskurs aktiv mitzugestalten.

Damit Medienpädagoginnen und Medienpädagogen zukünftig noch eindrucksvoller als Pionierinnen und Pioniere für die gemeinsame Idee zur freien Bildung und Openness fungieren können, erscheint es notwendig, dass sich OER als Standard in der medienpädagogischen Praxisarbeit etablieren. Dazu ist es wichtig, das Wissen und die Kompetenzen des Bildungspersonals im praktischen Umgang mit OER systematisch etwa in Form von Weiterbildungen zu erhöhen - wie z.B. hinsichtlich Lizensierung, Erstellung oder Verbreitung von OER. Ebenso wäre es wünschenswert noch mehr der bereits existierenden OER-Materialien im Kontext Medienpädagogik sichtbar zu machen und so die Lust, sich mit OER zu beschäftigen, bei den beteiligten Akteuren stärker zu fördern. 


\section{Literatur}

Baacke, Dieter. 1996. «Medienkompetenz - Begrifflichkeit und sozialer Wandel». In Medienkompetenz als Schlüsselbegriff, herausgegeben von Antje Von Rein, 112-24. Bad Heilbrunn: Klinkhardt.

Baethge, Martin. 2006. «Das deutsche Bildungs-Schisma: Welche Probleme ein vorindustrielles Bildungssystem in einer nachindustriellen Gesellschaft hat». SoFi-Mitteilungen (34): 13-27.

Bellinger, Franziska, und Kerstin Mayrberger. 2019. "Systematic Literature Review zu Open Educational Practices (OEP) in der Hochschule im europäischen Forschungskontext». MedienPädagogik: Zeitschrift für Theorie und Praxis der Medienbildung 34 (Research and OER): 19-46. https://doi.org/10.21240/ mpaed/34/2019.02.18.X.

BMZ. 2017. Der Zukunftsvertrag für die Welt. Die Agenda 2030 für nachhaltige Entwicklung. Herausgegeben vom V. für Bundesministerium für Wirtschaftliche Zusammenarbeit und Entwicklung. Bonn. http://www.bmz.de/de/mediathek/publikationen/reihen/infobroschueren_flyer/infobroschueren/Materialie270_zukunftsvertrag.pdf.

Bock, Annekatrin, und Maren Tribukait. 2019. «Kultur des Teilens: Ein kritischer Blick auf ein zentrales Konzept der OER-Bewegung». MedienPädagogik: Zeitschrift für Theorie und Praxis der Medienbildung 34 (Research and OER): 47-66. https://doi.org/10.21240/mpaed/34/2019.02.22.X.

Damnik, Gregor, Sebastian Horlacher, Anne Lauber-Rönsbach, und Hermann Körndle. 2018. «Lernen durch die Gestaltung von digitalen Medien - Warum offene Bildungsmaterialien einen Mehrwert bieten können». In Neues Handbuch Hochschullehre, herausgegeben von Brigitte Berendt, Birgit Szczyrba, Andreas Fleischmann, Niclas Schaper, und Johannes Wildt, 87, A. 3.34:41-62. Berlin: Raabe - Fachverlag für Wissenschaftsinformation.

Deimann, Markus. 2012. "Offene Bildung und offenes Lernen - mehr als nur eine Alternative für E-Learning». In Handbuch E-Learning, Expertenwissen aus Wissenschaft und Praxis, herausgegeben von Andreas Hohenstein und Karl Wilbers. Bd. 42. Köln: Wolters und Kluwers.

Deimann, Markus. 2018. Open Education: Auf dem Weg zu einer offenen Hochschulbildung. Bielefeld: Transcript Verlag.

Deimann, Markus, Jan Neumann, und Jöran Muuß-Merholz. 2015. «Whitepaper Open Educational Resources (OER) an Hochschulen in Deutschland. Bestandsaufnahme und Potenziale 2015». Herausgegeben von open-educationalresources.de - Transferstelle für OER. https://open-educational-resources.de/ wp-content/uploads/Whitepaper-OER-Hochschule-2015.pdf.

Echterhoff, Charlotte. 2012. «Potenziale Aktiver Medienarbeit für den Bildungsprozess». Ludwigsburger Beiträge zur Medienpädagogik 15: 1-8.

Erdsiek-Rave, Ute, und Marei John-Ohnesorg. 2014. Schöne neue Welt? Open Educational Resources an Schulen. Berlin: Friedrich-Ebert-Stiftung. http://library. fes.de/pdf-files/studienfoerderung/11147.pdf. 
Frey, Karl. 1982. Die Projektmethode. Weinheim: Beltz.

Hohenstein, Andreas, und Karl Wilbers. 2012. Handbuch E-Learning, Expertenwissen aus Wissenschaft und Praxis. Bd. 42. Köln: Wolters und Kluwers.

Horndasch, Sebastian, und Nils Weichert. 2014. "Creating Windows of Opportunities - Demokratisierung der Bildung durch Open Educational Resources». In Schöne neue Welt? Open Educational Resources an Schulen, herausgegeben von Ute Erdsiek-Rave und Marei John-Ohnesorg, 58-62. Berlin: FriedrichEbert-Stiftung. http://library.fes.de/pdf-files/studienfoerderung/11147.pdf.

Kerres, Michael. 2019. "Offene Bildungsressourcen und Open Education: Openness als Bewegung oder als Gefüge von Initiativen?» MedienPädagogik: Zeitschrift für Theorie und Praxis der Medienbildung 34 (Research and OER): 1-18. https://doi.org/10.21240/mpaed/34/2019.02.17.X.

Klafki, Wolfgang. 1996. Neue Studien zur Bildungstheorie und Didaktik. 5. Aufl. Weinheim/Basel: Beltz.

KMK Strategie. 2016. «Bildung in der digitalen Welt. Strategie der Kultusministerkonferenz». https://www.kmk.org/fileadmin/Dateien/pdf/PresseUndAktuelles/2017/Strategie_neu_2017_datum_1.pdf.

Koalitionsvertrag vom 12. März 2018. 2018. «Ein neuer Aufbruch für Europa. Eine neue Dynamik für Deutschland. Ein neuer Zusammenhalt für unser Land.» Koalitionsvertrag zwischen CDU, CSU und SPD 19. Legislaturperiode. Berlin. https://www.bundesregierung.de/resource/blob/975226/847984 /5b8bc2359od4cb2892b31c987ad672b7/2018-03-14-koalitionsvertrag-data. pdf?download=1.

Mayrberger, Kerstin. 2014. «Partizipativ Lehren und Lernen mit digitalen, freien Bildungsmaterialien - ein Beitrag aus mediendidaktischer Perspektive». In Schöne neue Welt? Open Educational Resources an Schulen, herausgegeben von Ute Erdsiek-Rave und Marei John-Ohnesorg, 51-57. Berlin: Friedrich-EbertStiftung. http://library.fes.de/pdf-files/studienfoerderung/11147.pdf.

Missomelius, Petra. 2019. "Theory And Policy - OER On Their Way Into Practice». MedienPädagogik: Zeitschrift für Theorie und Praxis der Medienbildung 34 (Research and OER): 90-100. https://doi.org/10.21240/mpaed/34/2019.03.01.X.

Muuß-Merholz, Jöran, und Felix Schaumberg. 2014. «Open Educational Resources (OER) für Schulen in Deutschland 2014. Whitepaper zu Grundlagen, Akteuren und Entwicklungen». Herausgegeben von Internet und Gesellschaft Collaboratory e.V. http://goo.gl/v657sa.

Rat für Kulturelle Bildung e.V. 2019. Jugend/YouTube/Kulturelle Bildung. Horizont 2019. Essen. https://www.rat-kulturelle-bildung.de/fileadmin/user_upload/ pdf/Studie_YouTube_Webversion_final_2.pdf.

Schell, Fred. 2003. Aktive Medienarbeit mit Jugendlichen. Theorie und Praxis. München: Kopaed. 
Schön, Sandra, Katharina Kreissl, Leonhard Dobusch, und Martin Ebner. 2017. Mögliche Wege zum Schulbuch als Open Educational Resources (OER). Eine Machbarkeitsstudie zu OER-Schulbüchern in Österreich. Herausgegeben von Forschungsbereich InnovationLab der Salzburg Research Forschungsgesellschaft m.b.H. Bd. 7. https://l3t.eu/oer/images/band15.pdf.

Stahl, Elmar. 2009. «Lernen durch Gestalten von digitalen Medien». In Lernchance Computer - Strategien für das Lernen mit digitalen Medienverbünden, herausgegeben von Rolf Plötzner, Timo Leuders, und Adalbert Wichert, 241-62. Münster: Waxmann.

UNESCO. 2019. «UNESCO-Empfehlung zu Open Educational Resources (OER). Verabschiedet auf der 40. Sitzung der UNESCO-Generalkonferenz». Übersetzung der Deutschen UNESCO-Kommission. Paris. https://www.unesco.de/sites/default/files/2019-11/UNESCO\%20Empfehlung\%2Ozu\%20OER.pdf.

Zauchner, Sabine, und Peter Baumgartner. 2007. «Herausforderung OER - Open Educational Resources». In Studieren neu erfinden - Hochschule neu denken, herausgegeben von Marianne Merkt, Kerstin Mayrberger, Rolf Schulmeister, Angela Sommer, und Ivo van den Berk, 244-52. Münster u.a.: Waxmann. https://www.pedocs.de/volltexte/2015/11329/pdf/Zauchner_Baumgartner_2007_ Herausforderung_OER.pdf. 